\title{
Measuring Temporal Variation in Presence During Game Playing
}

\author{
Jaeyong Chung* \\ School of Computer Science \\ The Australian National University
}

\author{
Henry J. Gardner ${ }^{\dagger}$ \\ School of Computer Science \\ The Australian National University
}

\begin{abstract}
A human-factors experiment has examined the effects of different sorts of technical anomalies, or breaks in presence(BIP), on user experience. Four different types of anomaly were investigated in an order-balanced experiment over two game types (violent versus non-violent game). A population of 36 volunteer participants was divided into two groups according to which game type they played first. After each game, participants were asked to rate the impact of the anomalies on their feelings of presence in the game experience and also of their effect on the recovery time by a video-cued slider rating method. There is evidence that participants feel different levels of impact and recovery from different sorts of technical anomalies. Most of all, the impact of BIP significantly depends on game mode; however there was no significant difference of recovery between the two groups. The additional results also indicate that BIP events occurred during the VR experience apart from those induced as part of the experimental design.
\end{abstract}

CR Categories: I.3.7 [Computer Graphics]: Three Dimensional Graphics And Realism-Virtual Reality

Keywords: computer game, presence, breaks in presence

\section{Introduction}

One of the markers of modern life is the ubiquity of video games as a favorite form of entertainment. With the latest technological advances, video games are metamorphosing from simple shoot-themup games into complex psychological experiences in which many gamers can compete and collaborate. Designing these complex experiences to be enjoyable and absorbing entertainment is an ultimate goal of game developers [Keeker et al. 2004].

In their study of increasingly sophisticated video games, some researchers have been investigating how the virtual reality concept of presence or "being there" can be utilized to enhance game experience (for example [McMahan 2003]). Presence is a complex, multi dimensioned construct and there are many factors which are known to potentially influence the feeling of presence [Ijsselsteijn et al. 2000; Schubert et al. 2001]. Numerous studies have examined the various influences on the feeling of presence (including [Lombard and Ditton 1997; Wilson et al. 2000; Murray et al. 2000; Wilson and Sasse 2000; Wang et al. 2006; Barfield et al. 2006; Khanna et al. 2006; Slater et al. 2009] ) and several measurement

*e-mail: jae.chung@anu.edu.au

†e-mail:henry.gardner@anu.edu.au approaches have been proposed [Lombard and Ditton 1997; IJsselsteijn et al. 1998; Witmer and Singer 1998; Slater and Steed 2000; Slater et al. 2006].

Some researchers have investigated the effects of playing "violent" video games on presence. Nowak et al. suggested that players who perceive a game to be more violent feel more presence than those who perceive less violence in the game [Nowak et al. 2008]. Tamborini et al. [Tamborini et al. 2004] found that a higher interactivity of game playing (in combat for example) was associated with a higher reported experience of presence. As another example, Ivory et al. [Ivory and Kalyanaraman 2007] examined the effects of violent content in games on the subjective feeling of presence, involvement, aggressive thoughts, aggressive feelings, and physiological arousal.

\subsection{Breaks in Presence (BIP)}

During game playing, a player is likely to experience various unexpected impediments or distractions which are known to degrade the feeling of presence. Such distractions or anomalies have been termed BIPs (Breaks in Presence) by Slater et al. [Slater and Steed 2000] who have used them to study the nature of the presence experience in virtual reality. Further, they have examined an association between a dramatic BIP (a screen whiteout) and physiological responses including skin conductance and heart rate [Slater et al. 2006].

Little attention has been given to recovery from BIPs. Based on a qualitative analysis from interviews, Garau et al. [Garau et al. 2008] suggested that participants have to make an effort to recover from BIPs, and that some of them required greater effort in order to experience presence again following BIPs. However, the authors did not provide a quantitative analysis of presence variation following BIPs and they did not consider interactions between the virtual environment content and the different sorts of BIPs.

In this paper we describe a user study which set out to examine how game players responded to induced technical anomalies in two categories of interactive games. One a non-violent game, was an exploration of a virtual world (the "navigation" game). The other was a game which was located in the same virtual world but which involved mortal combat (the "combat" game). We studied four types of BIP and, at the conclusion of each game, surveyed participants on the impact and ease of recovery from these BIPs. We used a method of analysis by a slider assessment which is relevant to investigating time-varying changes of the subjective feeling of presence.

\section{Measuring Presence}

The most common method for measuring presence is by means of post-experience questionnaires. Questionnaires are easy to produce and administer and are amendable to rapid statistical analysis. They are applicable to both interactive and non-interactive content. However, a post questionnaire cannot be used to rate the temporal variation of subjective presence.

To overcome this limitation, Ijsselsteijn et al. [IJsselsteijn et al. 1998] proposed the method of continuous rating using a hand-held
Permission to make digital or hard copies of part or all of this work for personal or classroom use is granted without fee provided that copies are not made or distributed for commercial advantage and that copies bear this notice and the full citation on the first page. Copyrights for components of this work owned by others than ACM must be honored. Abstracting with credit is permitted. To copy otherwise, to republish, to post on servers, or to redistribute to lists, requires prior specific permission and/or a fee Request permissions from Permissions Dept, ACM Inc., fax +1 (212) 869-0481 or e-mail permissions@acm.org.

VRCAI 2009, Yokohama, Japan, December 14 - 15, 2009.

(c) 2009 ACM 978-1-60558-912-1/09/0012 $\$ 10.00$ 

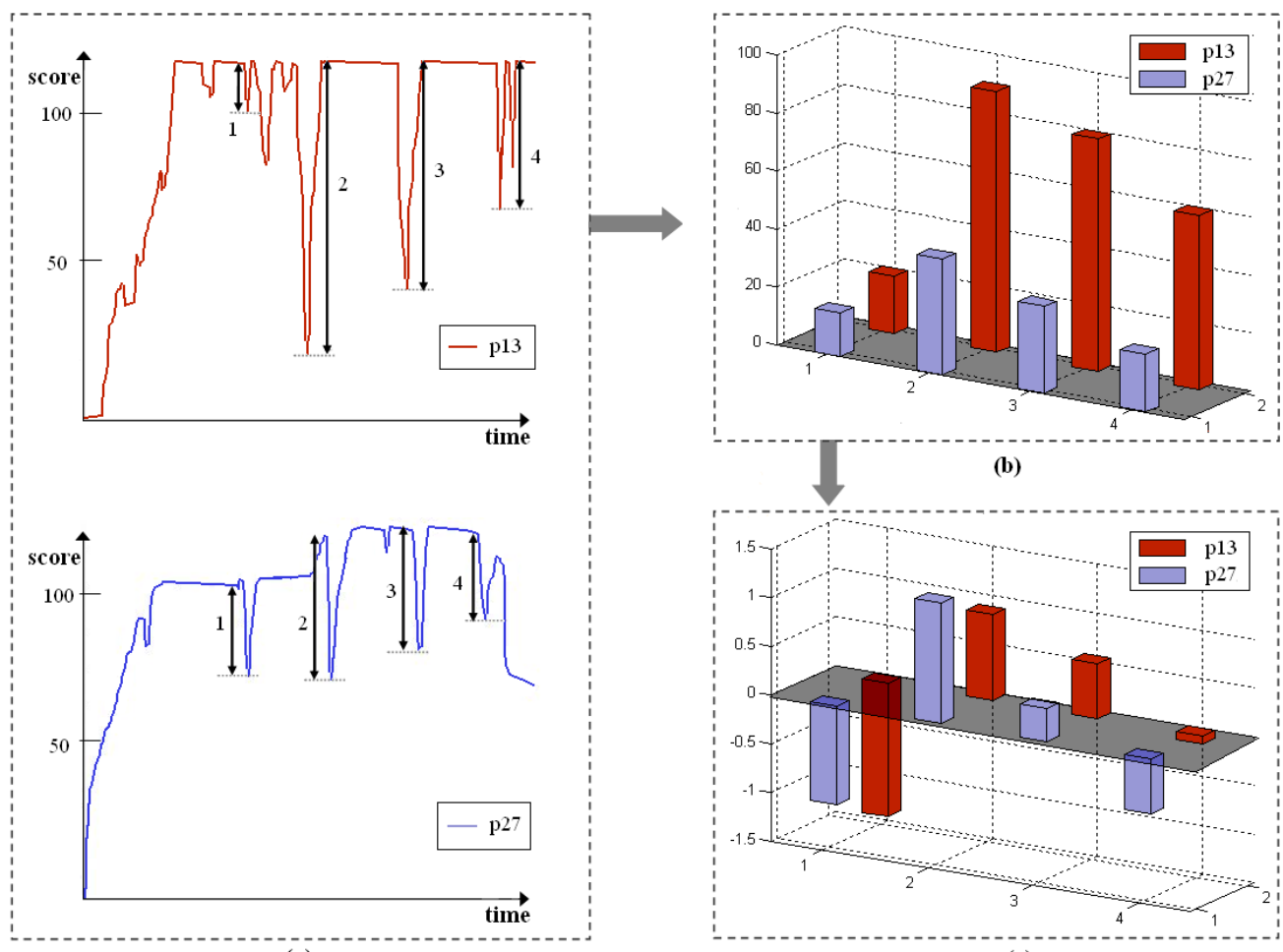

(a)

(c)

Figure 2: Standardizing two different scaled slider traces using z score transformation (a)the slider trace for p13 and p27. (b) measurements of the impact length from $(a)$. (c) the results of $z$-score transformation

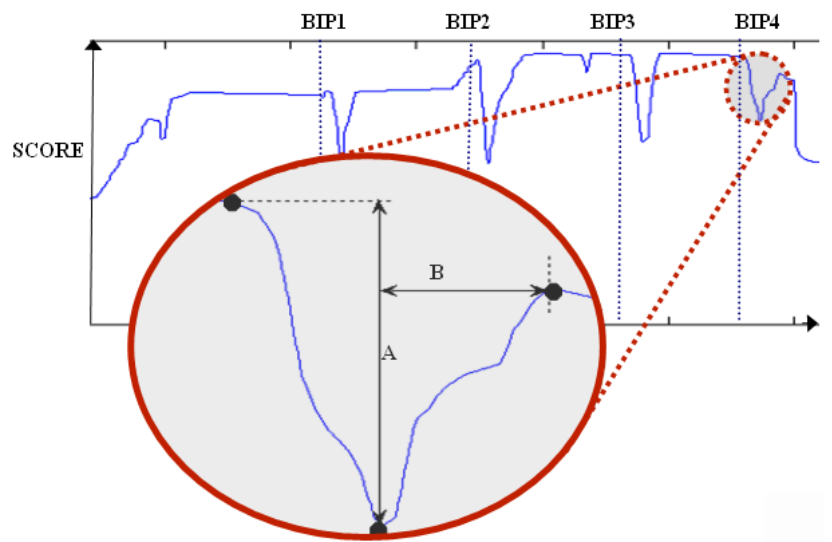

Figure 1: Measuring the impact and recovery phase of Breaks in Presence

slider. Their method provided a continuous presence rating as participants experienced non-interactive media. The participants' rating (the temporal variation in subjective feeling of presence) was logged against time. This promising methodology also had some crucial short-falls. The participants needed to manipulate a "real world" slider continuously during their presence experience, and this would have distracted their attention and effected the quality of the presence experience. Most of all, the nature of the Ijsselsteijn et al. [IJsselsteijn et al. 1998]'s experiments was restricted to noninteractive media, because one hand was used to operate the slider control.
An alternative approach to a slider to measure presence is to utilize physiological responses of participants such as Galvanic skin response, cardiovascular responses, ocular response, EEG and so on [Slater et al. 2006; Persky and Blascovich 2008; Laarni et al. 2003; Kallinen et al. 2007; Mikropoulos 2000; Schier 2000]. These methods produce a relatively continuous physiological data stream. Participants are not required to be involved in the measurement actively. However such responses can be influenced by many other unnecessary factors and they do not represent presence directly. For instance, skin response is known to be sensitive to arousal and heart rate is sensitive to hedonic valence which are important factors of emotion [Ijsselsteijn et al. 2000]. Since the feeling of presence is typically treated as a subjective factor, subtle or smooth presence variations would be difficult to measure directly using these techniques.

Garau et al. investigated how participants subjectively experienced BIPs by analyzing post-experience interviews [Garau et al. 2008]. An interesting aspect of their research was that participants were asked to draw a line representing the extent to their feeling of presence during a virtual reality experience. They investigated those hand-drawn graphs and classified them into four main patterns. They suggested that participants perceived induced whiteout anomalies as breaks in presence and, that these BIPs could have different intensity resulting in recovery time. In these studies the participants drew a graph at a given time without an absolute timeline.

\subsection{A new slider technique for measuring presence}

The main purpose of the present study is to investigate the temporal variation in subjective feeling of presence after BIPs. In attempting to overcome some limitations of previous measurements, we have 
adopted a "video-cued recall" based slider assessment method.

As described in 3.1 below, we chose a first person shooter (FPS) game for our research and modified it to have two different game modes (navigation and combat). During the experiment, the actual game playing of participants was recorded by video- capture software. Immediately after a game experience in a two-walled virtual reality (VR) theatre, the recording was then replayed inside the same VR theatre with participants assuming the same position as for the game but with the game controller replaced by a slider. The idea was that the high level of immersion associated with the VR theatre would help participants recall how they felt during the original game experience. During this assessment session, participants were asked to move the slider according to their level of presence (upward for higher presence and downward for lower presence). The slider measurements were logged as a function of time. For the slider assessments, we chose a commercial music fader and modified it to have force feedback on the slider control in order to avoid participants continuous checking its scale.

Data obtained were analyzed as shown in Figure 1. In this figure, A and $\mathrm{B}$ are the impact length and recovery time from a BIP. In order to estimate these values, we determined three inflection points immediately after a BIP. A Z score transformation was applied to these measurements of each participant (both impact length and recovery time) since they used different scales for their slider rating. The $\mathrm{Z}$ score transformation provides a way of standardizing data and allows comparison of observations from different normal distributions. Figure 2 demonstrates the effect of the standardization by z-score transformation. In this figure, P13 used a relatively larger scale during slider than P27. In contrast to previous work [IJsselsteijn et al. 1998] which applied this standardizing process to the entire data, we only considered the intensity and recovery value on the slider traces, because we were interested in the temporal variation in the short period immediately after a BIP.

\section{Experiment}

In order to investigate Breaks in Presence, we ran an experiment with 36 participants (undergraduate and postgraduate university students) with a mean age of 22 years (standard deviation of 2.9). All of the participants played computer games at least once a month. Recruitment was by advertisement around the campus and each participant received a movie ticket for their participation.

The experiment was conducted in a virtual reality theatre with two, rear-projected, $2.9 \mathrm{~m} \times 2.2 \mathrm{~m}$ screens, joined at an angle of $90 \mathrm{de}-$ grees and providing an almost 180 degree immersive field of view (see Figure 3). In this setting, we had full control over the ambient lighting and sound as well as the other programmable aspects of the virtual environment. Even though its stereoscopic and headtracking capabilities were not used, the large-display 3D graphics of this theatre was expected to increase the feeling of presence over playing games in a normal desktop setting. This study was approved by the human ethics committee of university and all participants were instructed according to the ethical guidelines.

\subsection{Game Environment}

We used a first person shooter (FPS) game which was originally written for a study of a commercial, online, 3D, game engine [Lee and Park 2004]. We ported this game to the two-screen display and modified one of its levels to implement the two different playing modes for our research.

Previously, studies of the effects of violent versus non-violent games compared different game environments selected from the

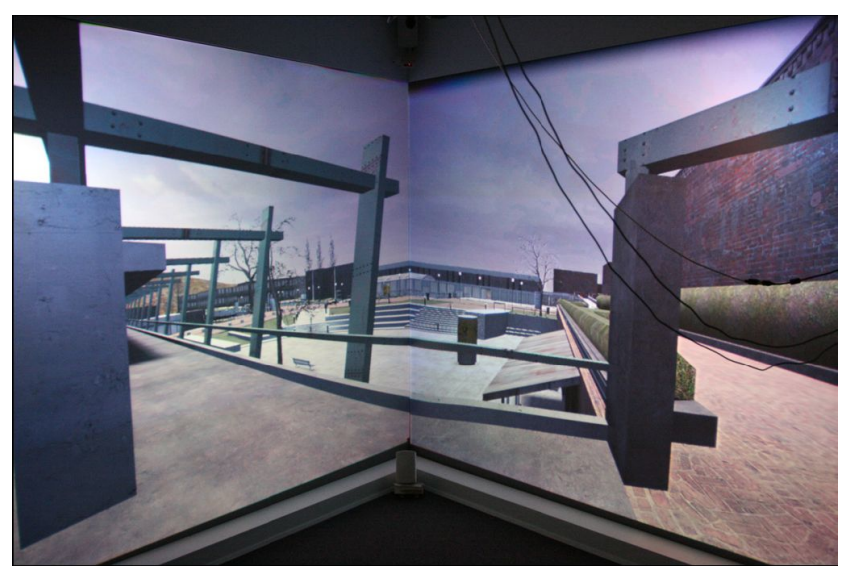

Figure 3: View of VR Theater

two genres [Nowak et al. 2008; Tamborini et al. 2004; Ivory and Kalyanaraman 2007]. In conducting our experiment, we wanted to avoid the influence of possible confounding factors such as differences in visual realism, the amount of 2D or 3D fidelity, interaction techniques, sound effects, technological advancement, and so on. Both game modes were therefore implemented in the one virtual environment.

Participants played two game modes: navigation and combat. In navigation mode, there was no particular mission and no threat of attack. Participants were told that they should explore the environment which was the interior of a two-story building including a grand staircase and balcony, all with medieval decor. Players had use of their weapon in this mode, and they could amuse themselves by taking shots at stationary enemy avatars which were scattered through the building but which would not retaliate.

In combat mode, participants were subject to continuous attack by enemies and they needed to engage in typical FPS tactics such as moving quickly, shooting, hiding and so on. The enemy avatars were under the control of a remote participant who was an experienced player of this game level and who tuned the intensity of their attack to be always just at, or above, the level of competence of the player.

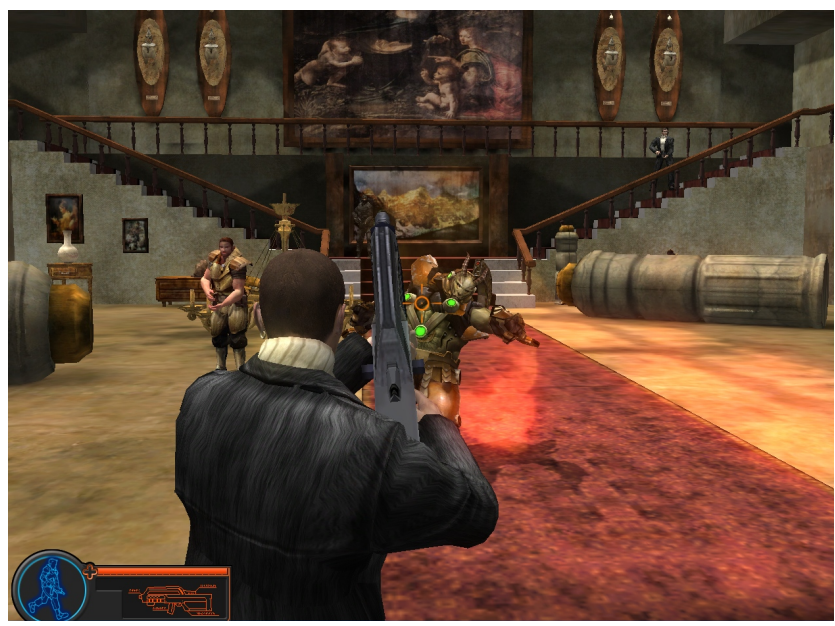

Figure 4: Screen shot of the game environment in combat mode 


\subsection{BIP design}

In order to investigate participants' subjective feeling of breaks in presence during game play, we induced four technical anomalies: low frame rate, sound absence, reverse control and screen blackout. Their details, and nomenclature, are given in Table 1. The criterion for selecting four BIP effects was carefully designed considering the theoretical determinants of presence [Ijsselsteijn et al. 2000; Schubert et al. 2001]], the likelihood of taking place in real game playing, and previous results related to "negative effects" to virtual experiences [Wang et al. 2006; Wilson and Sasse 2000; Murray et al. 2000; Slater et al. 2006; Claypool and Claypool 2007].

\begin{tabular}{|l|l|}
\hline Factor & Description \\
\hline \hline BIP1 (Low frame rate) & Degrade to 5fps(frame per second) \\
\hline BIP2 (Sound absence) & Sudden absence of sound \\
\hline BIP3 (Reverse control) & $\begin{array}{l}\text { Mouse and keyboard events were } \\
\text { inverted }\end{array}$ \\
\hline BIP4 (Black out) & Screen suddenly and totally black \\
\hline
\end{tabular}

Table 1: Breaks In Presence used in the experiment

Frame rate is determined by several conditions such as resolution and hardware configuration and it can change with what is currently happening during actual game playing. Previous work suggested that frame rate is closely related to game performance [Claypool and Claypool 2007]. For BIP1, considering several previous empirical studies related to frame rate, we choose 5 frame per second for inducing technical problem during game.

In BIP2, one sensory channel (audio) temporally cut out. This situation might be caused by bad headphone or speaker connection, sound card failure, conflict with other software applications and so on.

With BIP3, one of dominant factors that influences the sense of presence, "match between sensors and the display" [Ijsselsteijn et al. 2000] was destructively interfered. The mappings of mouse and keyboard controls to view control and movement were suddenly inverted to simulate a hypothetical situation where a player might fail to execute a control action correctly.

Similar to the BIP used by Slater et al. [Slater et al. 2006], BIP4 set the display to be totally black as if turn off the screen which believed to induce biggest impact and and recovery.

The four BIP anomalies were designed to be triggered at periodic intervals and each lasted for 3 seconds. In navigation mode, it was possible to be quite strict about the timing between each BIPs but in combat mode, other factors needed to be considered in order to have a realistic evaluation of the effect of each anomaly. For example, each BIP factor was not triggered in combat mode unless an enemy was located within the participants viewing frustum and the participant was engaged with that enemy. In the case of BIP3 (reverse control), the effect was not triggered unless a participants mouse or keyboard was active at the time. In fact, it turned out that the time differences between the triggering of events in navigation and in combat mode were small because of the continuous nature of enemy engagement in combat mode.

\subsection{Procedure}

Two groups of 18 participants were randomly assigned to two groups each of which played both game modes but in the opposite order. The Navigation Group played navigation mode first and combat mode second and Combat Group played combat mode first and navigation mode second. The order of BIPs in both game modes was randomly distributed.

At the beginning of the experiment, each participant received an information sheet about the experiment followed by a questionnaire related to their background, including age, gender, game experience. The information sheet was designed to explain the purpose of experiment, experiment procedure, game environment and the usage of equipment and so on. Most of all, participants might not be familiar with the concepts of presence and breaks in presence; therefore we more focused on this in the information sheet.

We explained that the game they were about to play was not a completed version and thus some technical problems, resulting in breaks in presence, would occur at any time. It was necessary to warn participants that anomalies would occur in order to attempt to normalize the impact of the first experience with subsequent experience.

Participants were provided with a training period (around four minutes) to practice the playing the game. This also allowed them to get used to the immersive virtual environment and adjust to the large field of view (FOV).

The actual experimental session took six minutes. Immediately after experiment session, participants were taken through a process of video-cued assessment of their game experience. Identical postexperiment surveys were administered after each game mode so that each group completed the survey twice(once for each game mode). Subsequently, participants were asked to complete Likertscaled questions concerning the overall presence, perceived impact and recovery for each BIP and so on.

\section{Results}

In this paper, we consider the first experience, between group, comparison, leaving the within group analysis and detailed treatment of the interview data for later work.

To investigate the effects of break in presence type and game mode on the severity of the break in presence as measured by the slider data multiple linear regression (MLR) models were fitted. These models used a measure of the severity of duration either the length for impact or the time for recovery (A and B in Figure 1) as the response variable and the following explanatory variables BIP type (low frame rate, sound absence, reverse control, and blackout) and game mode (navigation and combat). Interactions between BIP type and game mode were investigated.

As described in 2.1, the length and time variables were standardized using a $\mathrm{Z}$ score transformation. For each response value a suitable model was found by fitting a full model (a model that contained each BIP type, game mode and interactions between these two variables) and a backwards elimination type procedure based on the absolute value of the t-statistic. The backwards elimination stopped when all the variables remaining in the model had absolute values of the t-statistic that were greater than 1.96 in absolute value. One caveat was that if an interaction term was included in the model that the individual terms making up this interaction were included in the model regardless of their t-statistic values.

The first MLR looked at the effect of BIP type and game mode on the impact length. This model found a significant interaction between BIP1 (frame rate) and game mode (combat:BIP1, $p<0.01$ ) and between BIP3 (reverse control) and game mode (combat:BIP3, $p<0.05$ ). The estimated coefficients from the fitted model are:

These results suggest that the impact of BIP1 (frame rate) and BIP3 (reverse control) differ across game modes. The estimated effect 


\begin{tabular}{|c|c|c|c|}
\hline Term & Est. & SE & $p$-value \\
\hline \hline Intercept & -0.15 & 0.16 & 0.34 \\
\hline combat & -0.41 & 0.19 & 0.03 \\
\hline BIP1 & 0.17 & 0.25 & 0.49 \\
\hline BIP3 & -0.32 & 0.25 & 0.20 \\
\hline BIP4 & 0.77 & 0.19 & $5.75 \mathrm{e}-05$ \\
\hline combat:BIP1 & 0.91 & 0.32 & 0.005 \\
\hline combat:BIP3 & 0.75 & 0.32 & 0.02 \\
\hline
\end{tabular}

Table 2: Multiple linear regression for impact of BIPs

of BIP1 is $1.08(=0.91+0.17)$ in combat mode compared to 0.1681 in navigation mode and the estimated effect of BIP3 is $0.43(=0.75-$ 0.32 ) in combat mode compared to -0.32 in navigation model. Additionally, there is evidence that the impact of BIP4 (black out), while constant across modes, is different to the other breaks in presence.

The second MLR looked at the effect of BIP type and game mode on the recovery time. This model did not find any interactions or different effects across game modes. However, significant differences for BIP3 (reverse control, $p<0.05$ ) and BIP4 (black out, $p<0.01$ ) relative to BIP1 (frame rate) and BIP2 (sound absence) were found. The effects being constant across game modes. The estimated coefficients from the fitted model are:

\begin{tabular}{|c|c|c|c|}
\hline Term & Est. & SE & $p$-value \\
\hline \hline Intercept & -0.22 & 0.10 & 0.03 \\
\hline BIP3 & 0.43 & 0.17 & 0.01 \\
\hline BIP4 & 0.45 & 0.17 & 0.01 \\
\hline
\end{tabular}

Table 3: Multiple linear regression for recovery time for BIPs

From the two analyses it appears that game mode has impacts on length but not on the time. Further information about the impact of BIP and Mode on the impact length can be found by looking at the fitted values of length corresponding to the various levels of BIP and mode found in the rows of the table below. The column of fitted length values is the estimated length for the specified combination of BIP and mode.

\begin{tabular}{|c|c|c|c|}
\hline BIP & Mode & Fitted value & SE \\
\hline \hline BIP4 & Navigation & 0.62 & 0.16 \\
\hline BIP4 & Combat & 0.20 & 0.16 \\
\hline BIP1 & Navigation & 0.01 & 0.19 \\
\hline BIP1 & Combat & 0.51 & 0.19 \\
\hline BIP3 & Navigation & -0.47 & 0.19 \\
\hline BIP3 & Combat & -0.14 & 0.19 \\
\hline BIP2 & Navigation & -0.15 & 0.16 \\
\hline BIP2 & Combat & -0.57 & 0.16 \\
\hline
\end{tabular}

Table 4: Fitted values of the the length for impact

From these fitted values it can be seen that the combination of BIP4 (black out) and navigation result in the largest positive length, while the combination of BIP 2 (sound absence) and combat result in the largest negative length.

\section{Discussion}

The results of this study demonstrated that the impact of BIP1(low frame rate) and BIP3 (reverse control) is significantly higher in combat mode than in navigation mode. These two factors are more closely related to game interaction than sensory information. One possible reason for this result is that the nature of violent video games requires more active interactivity, therefore participants feel bigger impact from BIP1 and BIP3 during aggressive fighting. This suggests that the impact of a BIP factor is dependent on the nature of the virtual experience.

Contrary to our expectation, the results for the recovery time show that all other comparisons wash out in spite of some clear trends in the impact results. That is, recovery seems to depend on the type of BIP but seems to be independent of game type.

The impact of BIP4 (Black out) was expected to have the highest score in both game modes, but our results showed that BIP1 (Frame rate) scored higher than BIP4 in combat mode. This result might seem surprising considering a popular definition of presence as "perceptual illusion of non-mediation" [Lombard and Ditton 1997]. A possible explanation is that participants could maintain a state of high presence in spite of "turn off the screen" effect because they regarded this anomaly as a temporary problem which they believed would be soon fixed. By contrast, the sudden degradation of frame rate during aggressive game playing had a bigger negative impact on the participants sense of presence than did the the disruption of sensory information.

Participants' slider graphs showed several deep fluctuations at times when no deliberate BIP was occurring - there were various BIP factors during their VR experience other than the induced BIPs. Based on findings from the interviews and questionnaire results, participants described various instances when they felt breaks in presence, for instance, 'when killed by enemy' (the moment to be killed as well as to press keyboard button to revive), fallacious scene (broken texture etc), game imbalance (when realizing that an armored character has the same health points(HP) as an unarmored character) and so on. Based on these results, this work could be expanded by investigating more various BIP factors as well as including set of level in its duration and intensity. Further, we will take account of the potential impact of individual characteristics on presence such as gender, age, previous game experience and so on.

\section{Conclusion And Future Work}

The study reported here investigated how participants experienced intentional technical anomalies during game playing and compared the effect of different game contexts on the subjective feeling of presence. We also used a video-cued slider rating method for the continuous measurement of presence. This method can be a useful evaluation method within BIP related research because it is suitable for investigating sudden changes in the subjective feeling of presence quantitatively even during violent game play.

This study provides empirical evidence that participants feel different level of impact and recovery from different sorts of technical anomalies. Most of all, the impact of BIP significantly depended on game mode; however there was no significant difference in recovery between the two groups.

Games are increasingly being run on a range of platforms with different hardware, display size and computing power. Moreover new game technology allows users gesture and speech based interaction as well as wireless online game. The rapid pace of technological development can be expected to bring about many unforeseen technical issues. This research represents our first step toward understanding the variation of subjective presence as a consequence of negative technical effects. In order to minimize the intensity and recovery time for BIPs, we might be able to contrive new strategies of game design or modify software settings according to the hardware configuration or implement new technical effects which 
alleviate presence degradation. We believe that our research may contribute to the design of more enjoyable game experiences in the future.

\section{References}

Barfield, W., BAird, K. M., And Bjorneseth, O. J. 2006. Presence in virtual environments as a function of type of input device and display update rate. In Proceedings of the ACM Symposium on Virtual Reality Software and Technology.

Claypool, K., And Claypool, M. 2007. On frame rate and player performance in first person shooter games. In Multimedia Systems Journal (MMSJ), Springer, vol. 13, 3-17.

Garau, M., Friedman, D., Ritter Widenfeld, H., Antley., A., Brogni, A., AND Slater, M. 2008. Temporal and spatial variations in presence: Qualitative analysis of interviews from an experiment on breaks in presence. In Presence: Teleoperators and Virtual Environments, vol. 17.

IJsselsteijn, W., A, I., DE Ridder, H., HAmberG, R., Bouwhuis, D., AND Freeman, J. 1998. Perceived depth and the feeling of presence in 3dtv. In Displays, vol. 18, 207-214.

IJsselsteijn, W., A, I., De Ridder, H., Freeman, J., ANd AVONS, S. 2000. Presence: Concept, determinants and measurement. In Proceedings of the SPIE Human Vision and Electronic Imaging $V$.

IVory, J. D., And Kalyanaraman, S. 2007. The effects of technological advancement and violent content in video games on players' feelings of presence, involvement, physiological arousal, and aggression. In Journal of Communication, vol. 57, $532-555$.

Kallinen, K., Salminen, M., Ravaja, N., Kedzior, R., AND SAAKSJARVI, M. 2007. Presence and emotion in computer game players during 1st person vs. 3rd person playing view: evidence from self-report, eye-tracking, and facial muscle activity data. In Proceedings of the 10th International Workshop on Presence.

Keeker, K., Pagulayan, R. abd Sykes, J., and Lazarro, N. 2004. The untapped world of video games. In Conference on Human Factors in Computing Systems (CHI 2004).

Khanna, P., Yu, I., Mortensen, J., And Slater, M. 2006. Presence in response to dynamic visual realism: A preliminary report of an experiment study. In In Proceedings of the ACM Symposium on Virtual Reality Software and Technology, 364367.

LAARNI, J., RAVAJA, N., AND SAARI, T. 2003. Using eye tracking and psychophysiological methods to study spatial presence. In Proceedings of the 6th International Workshop on Presence.

LEE, H., AND PARK, T. 2004. Design and implementation of an online 3d game engine. In ICCSA 2004 : Lecture Notes In Computer Science.

Lombard, M., And DitTon, T. 1997. At the heart of it all: The concept of presence. In Journal of Computer MediatedCommunication, vol. 3.

McMahan, A. 2003. Immersion, engagement, and presence a method for analyzing 3-d video games. In The Video Game Theory Reader, Routledge, 67-86.
Mikropoulos, T. 2000. Brain activity on navigation in virtual environments. In Journal of Educational Computing Research, vol. 24, 1-12.

Murray, C. D., Arnold, P., And Thornton, B. 2000. Presence accompanying induced hearing loss: Implications for immersive virtual environments. In Presence: Teleoperators and Virtual Environments, vol. 9.

NowaK, K. L., Krcmar, M., And Farrar, K. M. 2008. The causes and consequences of presence: Considering the influence of violent video games on presence and aggression. In Presence: Teleoperators and Virtual Environments, vol. 17.

PERSKY, S., AND BlasCOVICH, J. 2008. Immersive virtual video game play and presence: Influences on aggressive feelings and behavior. In Presence: Teleoperators and Virtual Environments, vol. 17.

SCHIER, M. 2000. Changes in eeg alpha power during simulated driving: a demonstration. In International Journal of Psychophysiology, vol. 37, 155-162.

Schubert, T., Friedman, F., And Regenbrecht, H. 2001. The experience of presence: Factor analytic insights. In Presence: Teleoperators, and Virtual Environments, vol. 10.

Slater, M., AND SteEd, A. 2000. A virtual presence counter. In Presence: Teleoperators and Virtual Environments, vol. 9.

Slater, M., Guger, C., Edlinger, G., Leeb, R., Pfurtscheller, G., Antley, A., Garau, M., Brogni, A., Steed, A., And Friedman, D. 2006. Analysis of physiological responses to a social situation in an immersive virtual environment. In Presence: Teleoperators and Virtual Environments, vol. 15 .

Slater, M., Khanna, P., Mortensen, J., And Yu, I. 2009. Visual realism enhances realistic response in an immersive virtual environment. In IEEE Computer Graphics and Applications, vol. 2 .

TAmborini, R., Eastin, M. S., Skalski, P., Lachlan, K., FEDIUK, T. A., AND BRADY, R. 2004. Violent virtual video games and hostile thoughts. In Journal of Broadcasting and Electronic Media, vol. 48.

Wang, Y., Otitoju, K., LiU, T., Kim, S., and Bowman, D. A. 2006. Evaluating the effect of real world distractions on user performance in immersive virtual environments. In In Proceedings of the ACM Symposium on Virtual Reality Software and Technology.

WILson, G., AND SAsse, M. A. 2000. Investigating the impact of audio degradations on users: Subjective vs. objective. assessment methods. In Procs OZCHI'2000, 135-142.

Wilson, G., , Wilson, G. M., And SAsse, M. A. 2000. Do users always know what's good for them? utilising physiological responses to assess media quality. In In: The Proceedings of HCI 2000: People and Computers XIV?Usability or Else!, Springer, 327-339.

Witmer, B., AND Singer, M. 1998. Measuring presence in virtual environments: A presence questionnaire. In Presence: Teleoperators and Virtual Environments, vol. 7, 225-240. 